\title{
Héritages et renouveau urbain en Saxe
}

\section{Michel Deshaies}

\section{(2) OpenEdition \\ Journals}

Édition électronique

URL : http://journals.openedition.org/rge/2843

DOI : $10.4000 /$ rge.2843

ISSN : 2108-6478

Éditeur

Association des géographes de l'Est

Édition imprimée

Date de publication : 1 juin 2001

ISSN : 0035-3213

\section{Référence électronique}

Michel Deshaies, "Héritages et renouveau urbain en Saxe », Revue Géographique de l'Est [En ligne], vol. 41 / 3 | 2001, mis en ligne le 07 mars 2011, consulté le 23 septembre 2020. URL : http:// journals.openedition.org/rge/2843; DOI : https://doi.org/10.4000/rge.2843

Ce document a été généré automatiquement le 23 septembre 2020.

Tous droits réservés 


\title{
Héritages et renouveau urbain en Saxe
}

\author{
Michel Deshaies
}

1 Avec la réunification, les nouveaux Länder ont été soumis à un processus très brutal de transformation de l'économie et de la société. Il s'agissait de combler en quelques années les retards accumulés durant l'époque de la RDA : industrie peu productive aux équipements archaïques, productions inadaptées au marché, infrastructures de communication vétustes, villes aux centres historiques délabrés, environnement fortement pollué et dégradé. Au prix de transferts financiers gigantesques en provenance de l'Ouest, on a reconstruit un réseau de communication, développé une nouvelle industrie dont les équipements sont maintenant parmi les plus modernes d'Europe, réhabilité les quartiers centraux des villes.

2 C'est en effet dans les villes d'Allemagne de l'Est que l'on mesure le mieux l'ampleur des changements qui, en 10 ans, ont affecté le pays. Si, aujourd'hui encore, il subsiste bien des quartiers délabrés à proximité du centre de Leipzig, ou des ruines dans le centre de Dresde, ces traces du passé disparaissent peu à peu grâce à des opérations de réhabilitation, ou en cédant la place à des immeubles de verre abritant les nouvelles activités économiques, en plein essor depuis la réunification. Le renouveau économique qui a succédé à l'effondrement survenu au moment de la réunification affecte en effet principalement les grandes villes, points de convergence de l'essentiel des investissements. Or, en dehors de Berlin, le Land le plus urbanisé en Allemagne de l'Est est la Saxe, et c'est précisément dans ce «Freistaat Sachsen " que les évolutions observées depuis la réunification ont été les plus favorables.

3 «Land aux riches héritages » (F. Reitel, 1995), la Saxe semble en effet renouer avec un passé prestigieux. Bien dotée en ressources minières (argent des Monts métallifères), la Saxe resta pendant des siècles l'une des plus riches régions d'Allemagne. Les princes de Saxe tirèrent largement parti des richesses créées pour embellir leur capitale, Dresde, et en faire l'une des plus belles cités d'Europe, «la Florence de l'Elbe ». De son côté, Leipzig, idéalement placée au carrefour de la route Berlin-Munich avec celle joignant 
l'Europe rhénane à la Pologne, s'enrichissait par le commerce en devenant l'une des plus grandes villes de foire (Messestadt) d'Allemagne.

Sur ces bases, la Saxe devient au XIXe siècle le berceau de la révolution industrielle sur le continent, et c'est à Chemnitz que l'on fabrique par exemple les premières locomotives allemandes. Mais, contrairement à la Ruhr, l'industrialisation de la Saxe ne repose pas sur l'exploitation des richesses du sous-sol, les ressources charbonnières de cette région à longue tradition minière étant très limitées. Elle s'appuie sur les qualités d'une main-d'œuvre nombreuse et en forte croissance, puisque la population de la Saxe quadruple en un siècle pour atteindre près de 5 millions d'habitants à la veille de la Première Guerre mondiale. Cette main-d'œuvre est employée non seulement dans des industries traditionnelles comme le textile (Chemnitz est ainsi surnommée la Manchester saxonne), mais aussi dans de nouvelles industries à forte capacité d'innovation, développées par quelques inventeurs-entrepreneurs comme August Horch, un des fondateurs de l'industrie automobile allemande. Si la croissance se ralentit dans l'entre-deux-guerres et que les premiers signes de vieillissement de cette région précocement industrialisée apparaissent déjà, la Saxe reste néanmoins à la veille de la seconde guerre mondiale, l'une des deux grandes régions industrielles du pays. Ses deux villes principales, Leipzig (700 000 habitants en 1939) et Dresde (630 000 habitants en 1939) comptent alors parmi les plus grandes villes d'Allemagne (respectivement $5^{\mathrm{e}}$ et $7^{\mathrm{e}}$ villes du pays).

5 La guerre et ses destructions, les pertes territoriales qui la rejettent en situation périphérique et la division de l'Allemagne qui la coupe de ses relations avec la région rhénane, sapent les bases de sa prospérité. Mais ce sont surtout les choix économiques opérés durant les 40 ans de la RDA qui s'avèrent particulièrement négatifs pour l'avenir. Certes, dans le cadre du CAEM, l'industrie de la Saxe paraît être particulièrement performante, mais lorsqu'après la réunification elle est confrontée aux productions occidentales, elle s'effondre, victime d'une absence de modernisation et de l'inadaptation de ses productions au marché, à l'image de la Trabant désuette fabriquée à Zwickau. Ces réalités sont apparues dans toute leur ampleur après la réunification que les foules saxonnes, rassemblées en manifestations de masse sur l'Augustusplatz de Leipzig, durant l'automne 1989, ont fortement contribué à préparer.

6 Pour analyser les évolutions observées dans les villes et dresser une sorte de bilan, plus de 10 ans après la réunification, l'équipe rédactionnelle de la RGE a pensé qu'il fallait donner la parole à des géographes saxons dont les ouvrages et les articles sont souvent peu connus des lecteurs francophones. C'est ainsi que les géographes de l'université de Leipzig proposent aux lecteurs de la RGE un tableau riche et nuancé des transformations et des évolutions en cours en Saxe.

7 Le professeur Helga Schmidt nous montre ainsi l'ampleur de la désindustrialisation qu'a subi le Land et ses conséquences sociales et démographiques: l'importance du chômage et le recul de la population, en particulier dans les villes. Mais en même temps, elle identifie les signes d'espoir pour l'avenir, notamment grâce à l'importance des investissements consentis dans une nouvelle industrie qui, implantée par les konzerns de l'Ouest, renoue néanmoins avec les traditions industrielles de la région, la construction automobile par exemple. Ce renouveau, qui laisse à l'écart une bonne partie des régions frontalières, concerne essentiellement les grandes villes, entre lesquelles le Land cherche à développer les relations, afin de constituer une vaste région urbaine, le Triangle de Saxe. 
8 L'ampleur des transformations en cours depuis la réunification se traduit avec une acuité toute particulière dans les paysages urbains qui se sont métamorphosés, notamment dans les grandes villes comme Dresde et surtout Leipzig, dont le bâti des quartiers historiques du centre, négligé par les autorités de la RDA, était complètement délabré. Vera Denzer nous décrit ainsi le processus de rénovation/réhabilitation des centres des grandes villes saxonnes et étudie les stratégies suivies par ces villes pour redonner une attractivité à leur centre.

9 Si les centres des villes ont changé de visage, c'est peut-être dans les périphéries urbaines que l'on mesure le mieux l'ampleur des transformations. En effet, jusqu'en 1989, la politique urbaine qui prévalait en RDA avait freiné considérablement l'étalement des villes en concentrant la population dans d'immenses ensembles d'habitations collectives. Le professeur Reinhard Wießner montre que, depuis la réunification, les grandes villes de Saxe ont connu un processus de périurbanisation accéléré. Les communes périurbaines sont ainsi devenues le lieu d'accueil privilégié des habitants quittant les villes centres à la recherche de logements plus confortables. Aussi, à l'image de ce qui s'est passé trente ans plus tôt en RFA, les lotissements de maisons individuelles se sont multipliés, entraînant un étalement spatial très rapide des agglomérations, malgré le recul important de la population régionale.

D'une façon plus significative encore, Maike Hoppmann et Helga Schmidt montrent que ce sont non seulement les zones d'habitation, mais aussi les activités commerciales qui se sont implantées massivement dans l'espace périurbain. Suivant un processus de rattrapage accéléré du modèle occidental, les grands centres commerciaux se sont multipliés «auf der grünen Wiese». Mais plus encore qu'à l'Ouest, l'importance des grandes surfaces commerciales périphériques est devenue telle que les centres-villes risquent de perdre une grande partie de leur attractivité et de devenir des coquilles vides. Aussi tente-t-on de redonner à ces centres des fonctions permettant de préserver leur rôle traditionnel.

11 L'une des fonctions traditionnelles des villes, c'est de fournir des services et d'exercer ainsi une attraction sur un espace plus ou moins étendu en fonction de leur importance. Au temps de la RDA, les fonctions de services étaient, d'une manière générale, relativement peu développées, la mobilité de la population réduite et, en conséquence, les villes n'exerçaient pas une influence aussi importante qu'en RFA. Avec la réunification, le concept des lieux centraux, depuis longtemps en vigueur dans l'aménagement du territoire de la RFA, s'applique également dans les nouveaux Länder. Ulrich Knabe étudie la traduction concrète de ce concept dans l'ouest des Monts métallifères, une région de Saxe éloignée des grands centres urbains, et où aucune ville importante ne peut jouer seule le rôle de lieu central. D'où la volonté de développer un centre multipolaire capable de redonner du dynamisme à une région encore fortement marquée par les conséquences économiques et sociales de la réunification. À une échelle réduite, cet exemple résume d'ailleurs assez bien la situation des villes de Saxe dans le cadre allemand et européen.

Une fois passée la crise profonde qui a suivi la réunification, la Saxe est, certes, de tous les nouveaux Länder, celui qui a fait les progrès économiques les plus rapides. $\mathrm{Ce}$ renouveau de la Saxe, qui repose sur le développement d'une nouvelle industrie, s'enracine aussi dans les traditions industrielles de la région. Il s'observe surtout dans les grandes villes qui ont l'ambition de retrouver leur éclat d'antan, à l'image de Dresde, où l'on reconstruit la Frauenkirche, la plus belle église baroque d'Allemagne, 
détruite pendant les bombardements de 1945. Mais, ce renouveau économique et urbain a aussi ses limites, car Dresde, Leipzig et Chemnitz ne sont plus que l'ombre de ce qu'elles étaient avant la Seconde Guerre mondiale. Aujourd'hui, aucune n'atteint les 500000 habitants (Dresde a 472000 habitants, Leipzig 492 000, et Chemnitz 256000 en 2001, grâce d'ailleurs à l'absorption de communes voisines) et tant sur le plan démographique (Leipzig et Dresde sont désormais aux $14^{\mathrm{e}}$ et $15^{\mathrm{e}}$ rangs en Allemagne, derrière Nuremberg) que sur celui de l'économie, elles sont largement distancées par les grandes villes d'Allemagne de l'Ouest. Les foires de Leipzig par exemple, autrefois si importantes, arrivent très loin des chiffres de fréquentation de celles de Hanovre, Hambourg, Düsseldorf, Francfort ou Cologne.

13 Ainsi, si la nouvelle dynamique urbaine en cours en Allemagne de l'Est, et en particulier en Saxe, apparaît spectaculaire en ce qui concerne, par exemple, l'évolution des paysages urbains, il ne faut pas oublier que 40 ans de stagnation, voire de régression démographique et économique, ont lourdement obéré les chances des villes de l'Est de retrouver, individuellement, un rôle de premier plan en Allemagne. D'où la volonté affichée par le Land de développer les relations entre les grandes villes, afin de constituer une vaste région urbaine, capable de peser suffisamment dans le cadre européen ; c'est du moins l'ambition poursuivie à travers l'émergence du « Triangle de Saxe ».

\section{AUTEUR}

\section{MICHEL DESHAIES}

Université de Nancy 2 - 23, boulevard Albert- $\mathrm{I}^{\mathrm{er}}$ BP 33-97 54015 Nancy Cedex michel.deshaies@univ-nancy2.fr 